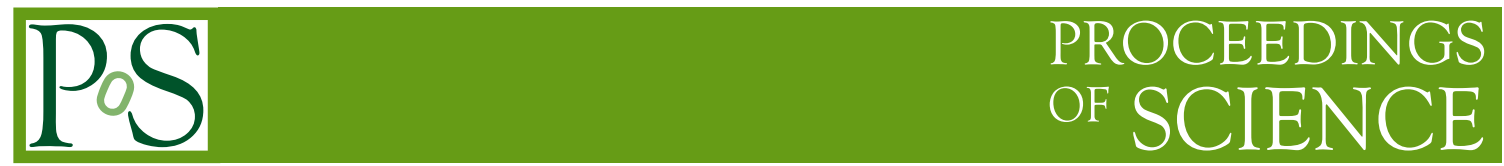

\title{
Glauber Gluons in SCET and the BFKL Equation
}

\author{
Sean Fleming ${ }^{* \dagger}$ \\ University of Arizona \\ E-mail: spe@email.arizona.edu
}

In this talk I discuss the importance of the Glauber region in factorization in general, and the connection of Glauber interactions in soft collinear effective theory (SCET) to the Regge region of QCD. I present results from my recent paper wherein I show how the Balitsky-Fadin-KuraevLipatov evolution equation arises out or a rapidity renormalization of the SCET Glauber interaction operator.

QCD Evolution 2015 -QCDEV2015-

26-30 mAY 2015

Jefferson Lab (JLAB), Newport News Virginia, USA

* Speaker.

${ }^{\dagger}$ A footnote may follow. 


\section{Introduction}

The principle of factorization underlies all theoretical predictions for hadronic processes. Simply put, factorization is the statement that short and long distances contributions to physical processes can be separated, up to corrections suppressed by powers of the relevant large scale in the process. The predictive power gained from this result stems from the fact that the incalculable long distance effects are universal and defined in an unambiguous way in terms of matrix elements. As a consequence, the non-perturbative long distance effects can be extracted in one process and then used in another. In general, proving factorization in QCD is a difficult task, and readers are referred to Refs. [1, 2, 3]) for reviews.

Soft collinear effective theory (SCET) $[4,5,6,7]$ was developed as an effective field theory (EFT) that would provide a framework for reformulating proofs of factorization in an operator formalism and for systematically treating power corrections. One consequence of an EFT approach is that it allows standard renormalization group techniques to be used for the resummation of double (Sudakov) logarithms as well as for single logarithms, which is often necessary for making perturbative calculations of high energy scattering events converge. A number of QCD factorization proofs were reproduced in Ref. [8] and subleading corrections were considered for a number of processes (see for example Refs. [9, 10, 11, 12, 13, 14, 15, 16, 17]).

One of the subtleties that makes proofs of factorization so difficult is the existence of the socalled Glauber region. Proofs of factorization rely on a set of powerful theoretical tools: power counting, pinch analysis via the Landau equations [18], and the Coleman-Norton Theorem [19]. The Landau equations allow for the isolation of pinch singularities which, via the Coleman-Norton Theorem can be identified with long-distance (infrared) physics. Generically pinch singularities can be identified with one of three momentum regions: collinear, soft, or Glauber. In the collinear region internal propagators become collinear with external particles, and in the soft region they become soft relative to external particles. In either of these limits particles can approximately stay on their mass shell. The Glauber region, however, is special as it corresponds to off-shell modes (Glauber modes) with $k_{\perp} \gg k^{+}, k^{-}$, which leads to a two-dimensional Coulomb-like interaction between and amongst collinear and soft particles [20,21]. The presence of Glauber interactions is problematic because they can destroy factorization [21, 22]. Fortunately, it has been shown that for sufficiently inclusive quantities the sum over final-state cuts cancels unwanted pinches, and thereby eliminates Glauber contributions [23, 24, 25].

SCET as it was originally formulated, however, did not include Glauber type interactions. An attempt to include Glauber interactions between collinear quarks moving in opposite directions in SCET was made Ref. [26] where factorization of the Drell-Yan cross section was reconsidered. Unfortunately, this attempt did not account for the overlap between different moment regions and failed as a result. The analysis was taken up in Ref. [27] where it was concluded that "for the exclusive Drell-Yan amplitude the correct effective theory would require Glauber modes." Though the authors did not consider under which circumstances the contribution from Glauber interactions cancel. In addition, a number of groups have considered the role of Glauber interactions between collinear and soft degrees of freedom in dense QCD matter [28, 29, 30].

A second, seemingly unrelated issue concerning the formulation of SCET was raised in Refs. $[31,32]$, where it was pointed out that Regge behavior appears to fall outside of the usual organizing 
scheme of SCET. Specifically, Regge behavior refers to the emergence of power-law behavior for scattering amplitudes. In perturbative QCD this arises out of a summation of ladder graphs which gives rise to the Balitsky-Fadin-Kuraev-Lipatov (BFKL) evolution equation [33, 34] (see Ref. [35] for a very readable treatment). The solution of the leading logarithmic (LL) BFKL equation gives the total cross section for high energy scattering with just such a power law form. Clearly, as an EFT of QCD at high energy SCET needs to be able to reproduce the BFKL results.

Recently there has been renewed activity in including the Glauber contribution in SCET: Stewart and Rothstein have presented preliminary results in formulating the operator basis for Glauber interactions [36], and Refs. [37, 38] showed how the Glauber contribution gives rise to Regge behavior. In this talk, after a brief review of SCET, I discuss the importance of the Glauber contribution in general and how Glauber interactions in SCET give rise to the BFKL equation.

\section{SCET: a brief review}

SCET describes the dynamics of highly energetic particles moving close to the light-cone interacting with a background field of soft quanta. The interaction of the collinear particle with the background introduces a small residual momentum component into the light-like collinear momentum so that collinear particles have momentum $p^{\mu}=Q n^{\mu}+p_{\perp}^{\mu}+k^{\mu}$ where $n^{\mu}=(1,0,0,-1)$. Momentum is split into the large component $Q$, the intermediate (soft) component $p_{\perp} \sim Q \lambda$, and the residual (ultra-soft or usoft) piece $k^{\mu} \sim Q \lambda^{2}$, with $\lambda \ll 1$ a small parameter. However, the interaction of a collinear particle with the soft background is not the only possibility. As shown in Fig. 1 a collinear quark (dashed line) can also radiate one (or more) collinear gluons (spring with line through it). These types of interactions are allowed since they do not require the gluon to have a large invariant mass: any collinear particle can decay into any number of collinear particles. As

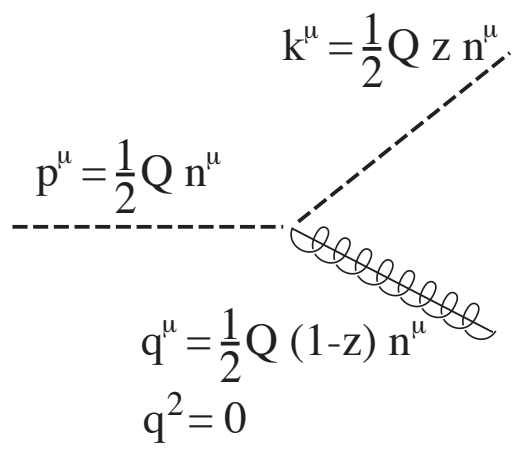

Figure 1: SCET collinear splitting allowed for any momentum fraction $z$.

a consequence the SCET Lagrangian consists of usoft, soft, and collinear sectors, and is systematically organized in powers of the small parameter $\lambda$. In Table 1 the effective theory quark and gluon fields are given along with their power counting in $\lambda$. The power counting is assigned such that in the action the kinetic terms for these fields are order $\lambda^{0}$. For instance, for an usoft gluon setting $\int d^{4} x_{u s} A_{u s} \partial^{2} A_{u s} \sim \lambda^{0}$ and using $d^{4} x_{u s} \sim \lambda^{-8}$ and $\partial^{2} \sim \lambda^{4}$ gives $A_{u s}^{\mu} \sim \lambda^{2}$. 


\begin{tabular}{cc|c|c} 
Type & Momenta $p^{\mu}=\left(p^{+}, p^{-}, p^{\perp}\right)$ & Fields & Field Scaling \\
\hline collinear & $p^{\mu} \sim\left(\lambda^{2}, 1, \lambda\right)$ & $\xi_{n, p}$ & $\lambda$ \\
& & $\left(A_{n, p}^{+}, A_{n, p}^{-}, A_{n, p}^{\perp}\right)$ & $\left(\lambda^{2}, 1, \lambda\right)$ \\
\hline \multirow{2}{*}{ soft } & $p^{\mu} \sim(\lambda, \lambda, \lambda)$ & $q_{s, p}$ & $\lambda^{3 / 2}$ \\
& & $A_{s, p}^{\mu}$ & $\lambda$ \\
\hline usoft & $k^{\mu} \sim\left(\lambda^{2}, \lambda^{2}, \lambda^{2}\right)$ & $q_{u s}$ & $\lambda^{3}$ \\
& & $A_{u s}^{\mu}$ & $\lambda^{2}$
\end{tabular}

Table 1: Power counting for the effective theory quarks and gluons.

To illustrate some important properties of SCET consider the quark sector of the Lagrangian. It can be split into two pieces: one coupling collinear to soft

$$
\mathscr{L}_{c s}=\bar{\xi}_{n, p^{\prime}} i n \cdot D \frac{\bar{\eta}}{2} \xi_{n, p}
$$

where $\xi_{n}$ is the collinear quark field, $\bar{n}=(1,0,0,1)$, and $i n \cdot D=i n \cdot \partial+g n \cdot A_{s}$. This expression looks very much like the HQET Lagrangian with the velocity $v^{\mu}$ replaced with the light-like vector $n^{\mu}$, and was first derived in Ref. [39]. The second piece of the collinear Lagrangian consists of interactions of only collinear particles among themselves

$$
\mathscr{L}_{c}=\bar{\xi}_{n, p^{\prime}}\left\{g n \cdot A_{n q}+i D_{c}^{\perp} \frac{1}{i \bar{n} \cdot D_{c}} i D_{c}^{\perp}\right\} \frac{\bar{n}}{2} \xi_{n, p} .
$$

The form of terms in the Lagrangian are restricted to this minimal set by the symmetries exhibited by SCET. These include invariance under separate collinear and soft gauge transformations [7], invariance under a global $U(1)$ helicity spin symmetry, and invariance under certain types of reparameterizations of the collinear sector of the Lagrangian [40, 41].

A remarkable consequence of the gauge symmetries of SCET is the factorization of (u)soft and collinear effects. For example, by introducing the usoft Wilson line

$$
Y(x)=\operatorname{Pexp}\left(i g \int_{-\infty}^{x} d s n \cdot A_{u s}(n s)\right)
$$

and redefine the collinear fields

$$
\xi_{n, p}=Y \xi_{n, p}^{(0)} \quad A_{n, q}^{\mu}=Y A_{n, q}^{(0) \mu} Y^{\dagger},
$$

the usoft gluons decouple from the collinear fields, i.e. $\mathscr{L}_{c s} \rightarrow 0$ in Eq. (2.1), and the collinear Lagrangian becomes independent of soft physics. At higher orders in the SCET expansion this decoupling does not occur, and factorization is broken. However, since higher order terms are suppressed breaking of the soft-collinear factorization is a small effect. This property of SCET allows us to obtain factorized forms of observables in a framework that is systematic.

A crucial aspect of separating usoft (or soft) and collinear degrees is the requirement that only one field has support in overlapping momentum regions. For example, collinear momentum in loop integrals may become usoft (while remaining collinear) which would overlap with the contribution 
of the usoft modes in the same loop integral. This leads to double counting, which must subtracted. If the fields in the Lagrangian are defined in a non-overlapping way this leads to the so-called zero-bin subtraction which removes any double counting [42].

We have not yet discussed the interaction of collinear particles with quanta having soft momentum scaling. An interaction between a soft particle and a collinear particle put the collinear particle offshell and so these types of interactions do not appear in the SCET Lagrangian. As a result soft particles can be trivially included in the theory by adding the Lagrangian for soft quarks and gluons with no interaction terms between soft and collinear. Typically we only need to consider SCET with collinear and usoft components (known as $\mathrm{SCET}_{\mathrm{I}}$ ), or with collinear and soft components (known as $\mathrm{SCET}_{\text {II }}$ ). There is an important difference $\mathrm{SCET}_{\mathrm{I}}$ and $\mathrm{SCET}_{\text {II }}$ which makes SCET II a much more challenging theory to work with. In $\mathrm{SCET}_{\mathrm{I}}$ there is a natural distinction between the collinear and usoft sectors since these modes have parametrically different invariant masses. In $\mathrm{SCET}_{\text {II }}$ there is no distinction between the invariant masses of the soft and collinear modes and they can be interchanged via boosts. The most important consequence of the latter property is that SCET $_{\text {II }}$ requires an additional rapidity regulator to maintain the independence of soft and collinear modes $[44,43]$ in the theory. Fig. 2 shows the momentum regions of $\mathrm{SCET}_{\mathrm{I}}$ and $\mathrm{SCET}_{\mathrm{II}}$, along with a mass-shell hyperbola for fixed invariant mass and fixed transverse momentum. The location of collinear, anti-collinear, and soft momentum scaling on the hyperbola is indicated. Usoft momentum is located in the lower left part of the graph and is clearly not on the same invariant-mass hyperbola as collinear momentum.

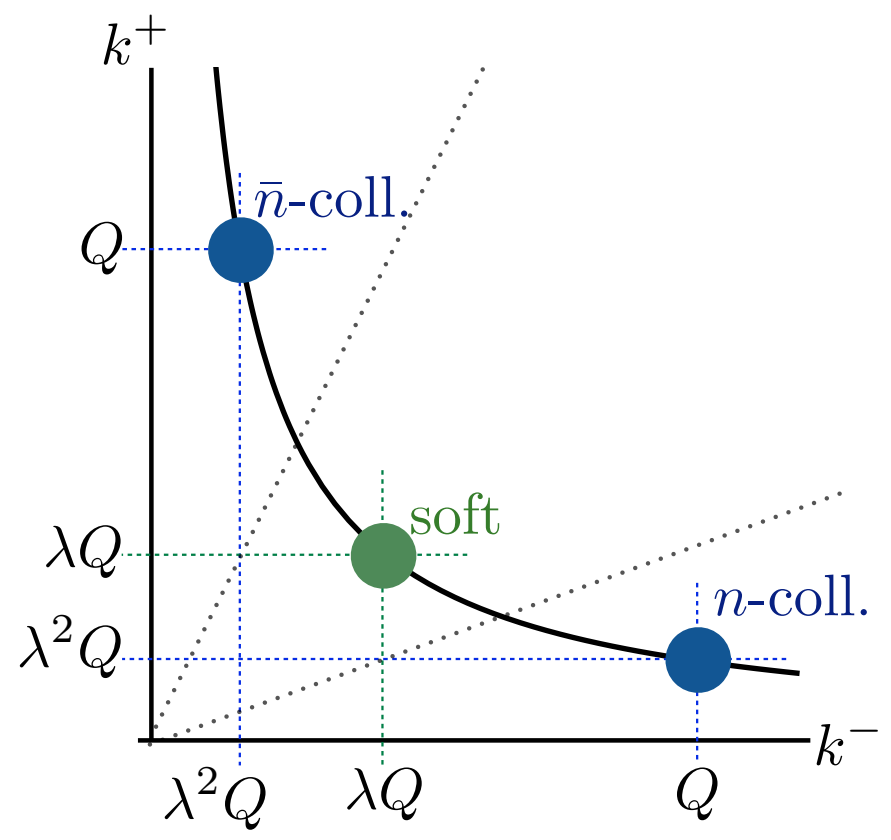

Figure 2: The momentum regions of $\mathrm{SCET}_{\mathrm{I}}$ and $\mathrm{SCET}_{\mathrm{II}}$, and a mass-shell hyperbola with fixed invariant mass. Collinear, anti-collinear and soft modes are indicated. Usoft modes sit in the lower left part of the plot. 


\section{The Glauber region}

The best way to understand the Glauber region is to consider the scattering of two high energy quarks moving in opposite directions $q\left(p_{1}\right)+q\left(p_{2}\right) \rightarrow q\left(p_{1}^{\prime}\right)+q\left(p_{2}^{\prime}\right)$ with large invariant mass $s=\left(p_{1}+p_{2}\right)^{2}$ and small momentum transfer $t=\left(p_{1}-p_{1}^{\prime}\right)^{2} \ll s$. The leading contribution is an exchange of an off-shell gluon between the quarks, resulting in a two-dimensional Coulomb like potential in transverse momentum. The diagram to consider is that in Fig. 3(a). Take all quarks to

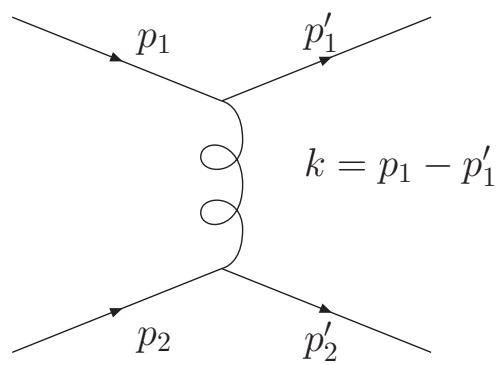

(a)

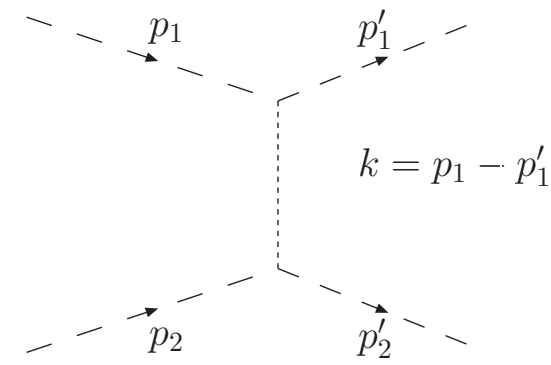

(b)

Figure 3: Leading order contribution to forward quark-quark scattering at high energy: (a) QCD diagram, (b) SCET diagram (dashed lines indicate collinear quarks, and dotted lines Glauber gluons).

be massless and on-shell, and let the momentum $\vec{p}_{1}$ define the $z$-axis. Then, the incoming momentum can be expressed in terms of two light-like vectors $n^{\mu}=(1,0,0,1)$ and $\bar{n}^{\mu}=(1,0,0,-1)$ :

$$
p_{1}^{\mu}=\frac{\sqrt{s}}{2} n^{\mu} \quad p_{2}^{\mu}=\frac{\sqrt{s}}{2} \bar{n}^{\mu} .
$$

The outgoing momentum can be expressed in a Sudakov decomposed form as well:

$$
\begin{aligned}
& p_{1}^{\prime \mu}=\frac{1}{2}(\sqrt{s}-\bar{n} \cdot k) n^{\mu}-\frac{1}{2} n \cdot k \bar{n}^{\mu}-k_{\perp}^{\mu} \\
& p_{2}^{\prime \mu}=\frac{1}{2} \bar{n} \cdot k n^{\mu}+\frac{1}{2}(\sqrt{s}+n \cdot k) \bar{n}^{\mu}+k_{\perp}^{\mu} .
\end{aligned}
$$

The outgoing quarks are taken to be on-shell so they must have

$$
n \cdot k=\frac{-\vec{k}_{\perp}^{2}}{\sqrt{s}-\bar{n} \cdot k} \quad \bar{n} \cdot k=\frac{\vec{k}_{\perp}^{2}}{\sqrt{s}+n \cdot k} .
$$

In the forward region we have $k^{2}=n \cdot k \bar{n} \cdot k+\vec{k}_{\perp}^{2}=t$ so that $k_{\perp}^{\mu} \sim \sqrt{t}$ and the above equation implies $n \cdot k \sim \bar{n} \cdot k \sim t / \sqrt{s} \ll k_{\perp}^{\mu}$. In this region the out-going momenta reduce to

$$
p_{1}^{\prime \mu} \approx \frac{\sqrt{s}}{2} n^{\mu}+\frac{\vec{k}_{\perp}^{2}}{2 \sqrt{s}} \bar{n}^{\mu}-k_{\perp}^{\mu} \quad p_{2}^{\prime \mu} \approx \frac{\vec{k}_{\perp}^{2}}{2 \sqrt{s}} n^{\mu}+\frac{\sqrt{s}}{2} \bar{n}^{\mu}+k_{\perp}^{\mu},
$$


and the invariant mass of the exchanged gluon is $k^{2} \approx-\vec{k}_{\perp}^{2}$, which gives rise to a two-dimensional coulomb-like interaction between the collinear quarks.

The presence of Glauber interactions is problematic because they can destroy factorization [21, 22], though for the right variables and for sufficiently inclusive quantities the sum over final-state cuts cancels unwanted Glauber contributions [23, 24, 25]. However, it has recently been pointed out by Gaunt [45] that even for sufficiently inclusive observables there are some variables where Glauber contributions do not vanish. In particular, for hadronic transverse energy $E_{T}$ in hadronhadron collisions, the classic Collins-Soper-Sterman argument for the cancellation of Glauber gluons breaks down at the level of two Glauber gluons exchanged between the spectators.

In fact the presence of Glauber interactions can be seen when comparing calculations that assume cancellation of Glauber contributions with (data tuned) Montecarlo studies. Ref [46] studied the effect of underlying events (UEs) on the hadron-level transverse-energy distribution in Higgs boson production at the LHC at 8 and $14 \mathrm{TeV}$. The UE is thought to arise due to secondary multiple interactions between the colliding hadrons, and is presumably primarily due to Glauber exchange. The authors find that "the effect of the UE on the $E_{T}$ distributions is severe, making them much broader and moving the peak to much higher values of $E_{T}$." Fig. 4 are plots from Ref [46] which show the differential in $E_{T}$ cross section for Higgs production at the LHC for different hadronic transverse momentum cuts (histograms) along with the prediction that assumes cancellation of Glauber contributions (red curve). These plots clearly demonstrate that UEs are important on even
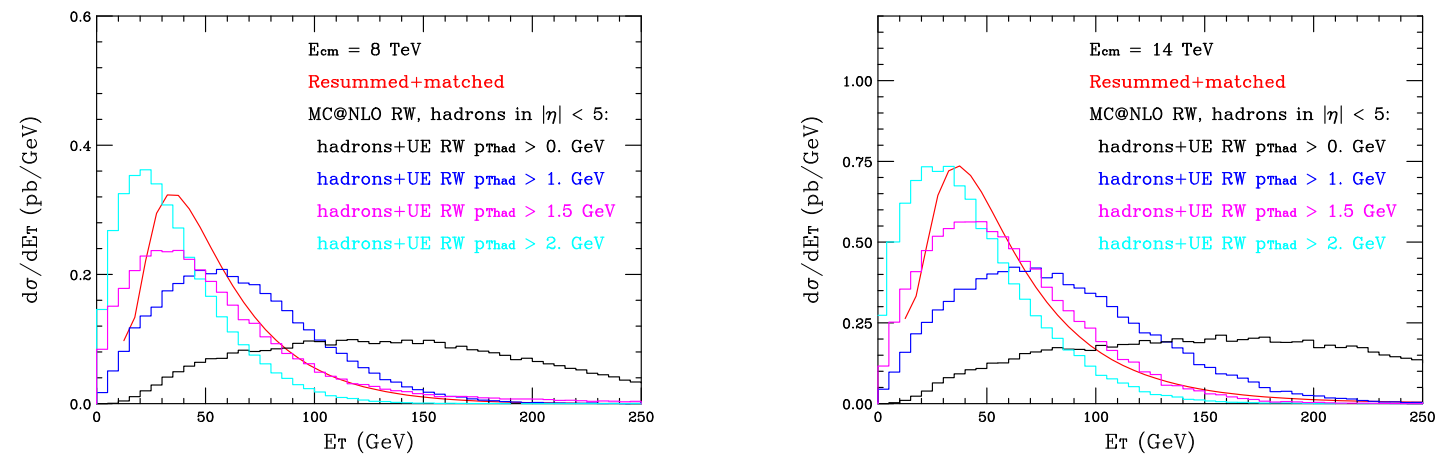

Figure 4: Hadron-level transverse-energy distribution in Higgs boson production at the LHC at 8 and 14 $\mathrm{TeV}$, including the effect of the underlying event. Red: resummed and matched to NLO, for comparison. Reweighted aMC@NLO+Herwig++ events with hadron maximum pseudorapidity $\eta^{c}=5$ : Black: $p_{T}^{c}=0$ GeV, Blue: $p_{T}^{c}=1.0 \mathrm{GeV}$, Magenta: $p_{T}^{c}=1.5 \mathrm{GeV}$, Cyan: $p_{T}^{c}=2.0 \mathrm{GeV}$.

a qualitative level, and provide a compelling reason to determine if the UEs are in fact due to multiple Glauber interactions. Clearly this makes a deeper understanding of the Glauber region an imperative.

\section{Glauber interactions and the BFKL equation}

To see how $\mathrm{SCET}_{\text {II }}$ gives the BFKL equation we need to determine the SCET operator that 
gives the leading part of the high-energy forward scattering process in Fig. 3(a). To see how such an operator arises in SCET we start with QCD and match onto SCET degrees of freedom. We carry out the matching depicted in Fig. 3 by expanding the QCD amplitude in the forward region

$$
\mathscr{A}_{Q C D}=-\frac{g^{2}}{\vec{k}_{\perp}^{2}} \bar{u}\left(p_{1}^{\prime}\right) T^{a} \gamma^{\mu} u\left(p_{1}\right) \bar{u}\left(p_{2}^{\prime}\right) T^{a} \gamma_{\mu} u\left(p_{2}\right) \approx-\frac{n \cdot \bar{n} g^{2}}{\vec{k}_{\perp}^{2}} \bar{\xi}_{n} T^{a} \frac{\hbar}{2} \xi_{n} \bar{\xi}_{\bar{n}} T^{a} \frac{h}{2} \xi_{\bar{n}},
$$

where $\xi_{n}$ and $\xi_{\bar{n}}$ are the high-energy limit of the QCD spinors for quarks moving in the $n^{\mu}$ and $\bar{n}^{\mu}$ direction respectively. This amplitude is reproduced by the SCET operator first derived in Ref. [47]

$$
\mathscr{O}_{G}^{n \bar{n}}=-\frac{2 g^{2}}{\vec{k}_{\perp}^{2}} \bar{\xi}_{p_{1}^{\prime}, n} T^{a} \frac{\hbar}{2} \xi_{p_{1}, n} \bar{\xi}_{p_{2}^{\prime}, \bar{n}} T^{a} \frac{h}{2} \xi_{p_{2}, \bar{n}},
$$

where $\xi_{p_{1}, n}$ and $\xi_{p_{2}, \bar{n}}$ are SCET quark fields. This operator is not gauge invariant under separate gauge transformations in the $n$ and $\bar{n}$ sectors, but can be made so by adding the appropriate SCET collinear Wilson lines [6]

$$
W_{n}=\sum_{\text {perms }} \exp \left(-\frac{g}{\bar{n} \cdot \mathscr{P}} \bar{n} \cdot A_{q, n}\right) \quad \text { and } \quad W_{\bar{n}}=\sum_{\text {perms }} \exp \left(-\frac{g}{n \cdot \mathscr{P}} n \cdot A_{q, \bar{n}}\right) .
$$

In addition, soft gluons with momentum that scales as $k_{s}^{\mu} \sim \sqrt{t}$ can be radiated from the collinear quarks. While such an interaction puts the collinear quark off-shell, it is order one in the power counting and must be summed into a soft Wilson line [7]

$$
S_{n}=\sum_{\text {perms }} \exp \left(\frac{-g}{n \cdot \mathscr{P}} n \cdot A_{s, q}\right) \quad S_{\bar{n}}=\sum_{\text {perms }} \exp \left(\frac{-g}{\bar{n} \cdot \mathscr{P}} \bar{n} \cdot A_{s, q}\right) .
$$

Including both collinear and soft Wilson liens we arrive at the $n-\bar{n}$ collinear Glauber operator

$$
\mathscr{O}_{G}^{n \bar{n}}=-8 \pi \alpha_{s}(\mu) \bar{\xi}_{p_{2}^{\prime}, \bar{n}} W_{\bar{n}} Y_{\bar{n}}^{\dagger} T^{a} \frac{h}{2} Y_{\bar{n}} W_{\bar{n}}^{\dagger} \xi_{p_{2}, \bar{n}} \frac{1}{\vec{P}_{\perp}^{2}} \bar{\xi}_{p_{1}^{\prime}, n} W_{n} Y_{n}^{\dagger} T^{a} \frac{\hbar}{2} Y_{n} W_{n}^{\dagger} \xi_{p_{1}, n}
$$

There are also collinear-soft Glauber operators which were considered in detail in Refs. [28, 29, 30], however, they are not needed here.

Next, we renormalize the operator in Eq. (4.5). The diagrams that contribute are shown in Fig. 5. The double lines in the diagrams in $(a)$ indicate that a soft gluon is emitted from one of the soft Wilson lines. The diagrams in $(a)$ are ultraviolet (UV) finite, but contain a rapidity divergence. The diagrams in (b) (plus a ghost-loop that is not shown) are UV divergent but do not have a rapidity divergence and are not needed in obtaining the BFKL equation. Thus we focus on the diagrams in Fig 5(a), which when summed give

$$
\mathscr{A}=-8 \pi \alpha_{s}(\mu) \bar{\xi}_{n} T^{a} \frac{\hbar}{2} \xi_{n} \bar{\xi}_{\bar{n}} T^{a} \frac{h}{2} \xi_{\bar{n}}\left[i N_{c} \alpha_{s}(\mu) \mathscr{I}\left(\vec{k}_{\perp}\right)\right]
$$

where

$$
\mathscr{I}\left(\vec{k}_{\perp}\right)=\int \frac{d q^{-}}{q^{-}} \int \frac{d^{2} q_{\perp}}{(2 \pi)^{2}} \frac{1}{\vec{q}_{\perp}^{2}} \frac{1}{(\vec{q}+\vec{k})_{\perp}^{2}} .
$$

In obtaining the expression in Eq. (4.6) a symmetry factor of one-half needs to be included as the first two diagrams in Fig 5(a) are identical to the second two diagrams. The integral over $q^{-}$results 

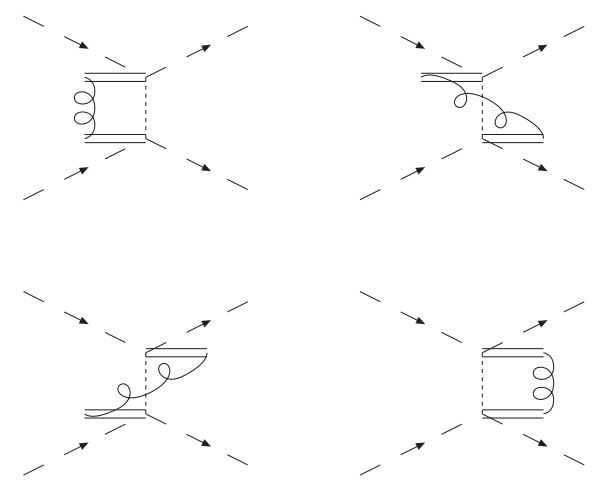

(a)
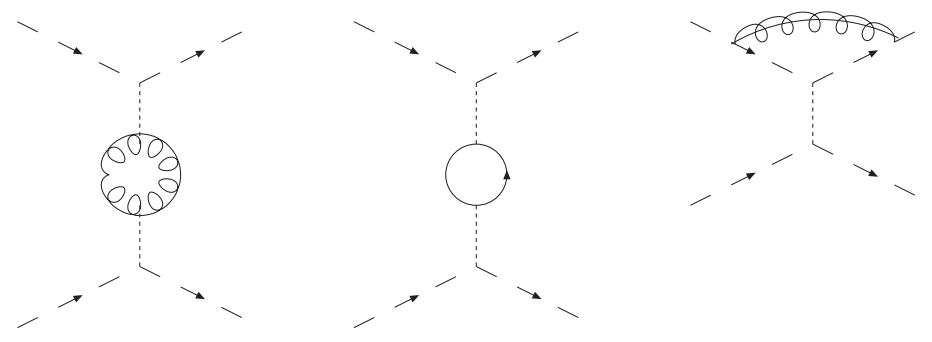

(b)

Figure 5: One loop Feynman diagrams contributing to the renormalization of $\mathscr{O}_{G}^{n \bar{n}}$. The double line in the diagrams in $(a)$ indicate soft gluon emission from a Wilson line. These diagrams have a rapidity divergence which gives the gluon Regge trajectory. The diagrams in $(b)$ have no rapidity divergence, but have UV divergences. The first two diagrams involve soft gluons and soft quarks (the soft-ghost loop diagram is not shown), and the UV divergence in these diagrams is cancelled by a soft Lagrangian counter-term. The last diagram involves the exchange of a collinear gluon (spring with a line) and the UV divergence is cancelled by a collinear Lagrangian counter-term.

in a rapidity divergence, while the integral over $q_{\perp}$, which in the literature is called the gluon Regge trajectory, contains IR divergences. To evaluate this integral we will need to introduce regulators for both types of divergences. Here we will regulate the rapidity divergence using the methods developed in Ref. [44, 43], and use a gluon mass (or dimensional regularization) to regulate IR divergences. With these modifications the integral above becomes

$$
\begin{aligned}
\mathscr{I}\left(\vec{k}_{\perp}\right) & =v^{2 \eta} w(v)^{2} \int \frac{d^{4} q}{(2 \pi)^{4}} \frac{1}{q^{-}} \frac{1}{q^{+}} \frac{\left(q^{3}\right)^{-2 \eta}}{q^{2}-m_{g}^{2}} \frac{1}{(\vec{q}+\vec{k})_{\perp}^{2}+m_{g}^{2}} \\
& =-i \frac{v^{2 \eta} w(v)^{2}}{\eta} \frac{\Gamma\left(\frac{1}{2}-\eta\right) \Gamma(1+\eta)}{(4 \pi)^{2} \sqrt{\pi}} \frac{1}{\left(k_{\perp}^{2}\right)^{1+\eta}} \int_{0}^{1} d x \frac{x^{\eta}}{\left[x(1-x)+m_{g}^{2} / k_{\perp}^{2}\right]^{1+\eta}} \\
& \approx \frac{-2 i}{(4 \pi)^{2}} \frac{w(v)^{2}}{\vec{k}_{\perp}^{2}}\left[\frac{1}{\eta} \ln \left(\frac{\vec{k}_{\perp}^{2}}{m_{g}^{2}}\right)+\ln \left(\frac{\vec{k}_{\perp}^{2}}{4 v}\right) \ln \left(\frac{\vec{k}_{\perp}^{2}}{m_{g}^{2}}\right)-\frac{1}{4} \ln ^{2}\left(\frac{\vec{k}_{\perp}^{2}}{m_{g}^{2}}\right)+i \pi \ln \left(\frac{\vec{k}_{\perp}^{2}}{m_{g}^{2}}\right)\right],
\end{aligned}
$$


where $w(v)$ is a bookkeeping parameter that has been introduced for convenience in deriving the rapidity RGE, and will eventually will be set to one [44, 43]. The rapidity divergence corresponds to the term that diverges as $\eta \rightarrow 0$. This rapidity pole must be subtracted by a rapidity counter-term. However, as the rapidity divergent term contains IR divergences a sensible rapidity RGE can not be derived. This issue is fixed if we consider forward scattering and include real emission diagrams.

The emission of a real soft gluon can occur from any of the soft Wilson lines as shown in Fig. 6(a) or from the exchanged Glauber gluon as shown in Fig. 6(b). The amplitude for the sum
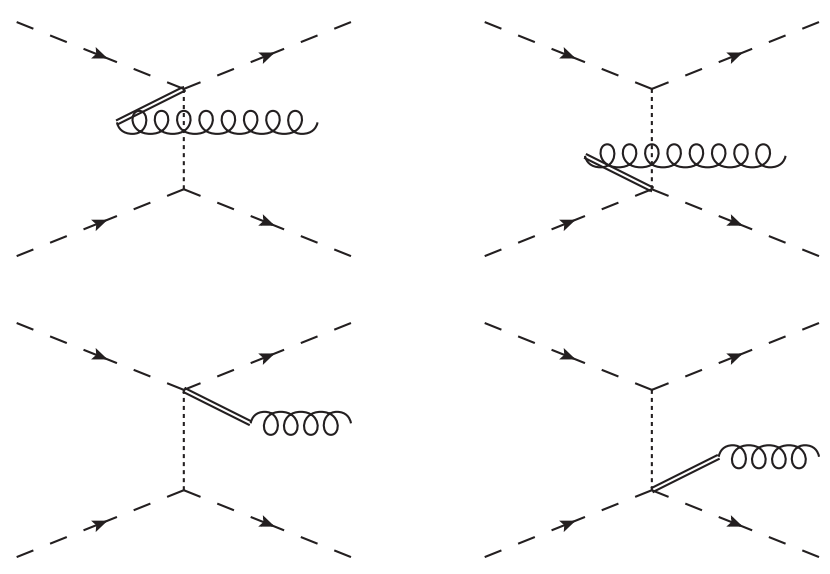

(a)

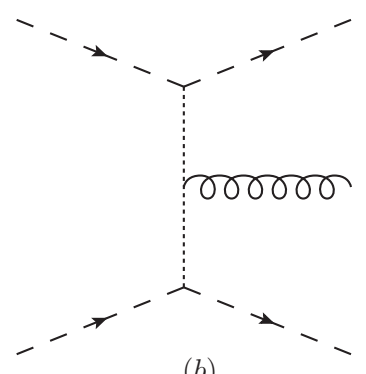

(b)

Figure 6: Real emission of soft gluons from the $n-\bar{n}$ Glauber interaction: (a) emission from the soft Wilson lines, (b) emission from the Glauber gluon.

of the four diagrams in Fig. 6(a) is

$$
\sum_{i=1}^{4} \mathscr{A}_{\text {real }}^{i}=-2 g^{2} \frac{1}{\vec{k}_{\perp}^{2}} \frac{1}{\vec{k}_{\perp}^{\prime 2}} \bar{\xi}_{n} T^{a} \frac{\hbar}{2} \xi_{n} \bar{\xi}_{\bar{n}} T^{b} \frac{h}{2} \xi_{\bar{n}}\left(-i g f^{a b c}\right)\left(\frac{n^{\alpha}}{n \cdot k^{\prime}} \vec{k}_{\perp}^{2}+\frac{\bar{n}^{\alpha}}{\bar{n} \cdot k} \vec{k}_{\perp}^{2}\right)
$$

and the amplitude for the diagram in Fig. 6(b) is

$$
\mathscr{A}_{\text {real }}^{5}=-2 g^{2} \frac{1}{\vec{k}_{\perp}^{2}} \frac{1}{\vec{k}_{\perp}^{\prime 2}} \bar{\xi}_{n} T^{a} \frac{\hbar}{2} \xi_{n} \bar{\xi}_{\bar{n}} T^{b} \frac{h}{2} \xi_{\bar{n}}\left(i g f^{a b c}\right)\left(k_{\perp}^{\alpha}+k_{\perp}^{\prime \alpha}-\frac{1}{2} \bar{n}^{\alpha} n \cdot k^{\prime}-\frac{1}{2} n^{\alpha} \bar{n} \cdot k\right)
$$


where the soft gluon momentum is $q^{\mu}=k^{\mu}-k^{\prime \mu} \approx \frac{1}{2} \bar{n} \cdot k n^{\mu}-\frac{1}{2} n \cdot k^{\prime} \bar{n}^{\mu}+\left(k_{\perp}-k_{\perp}^{\prime}\right)^{\mu}$. Adding these up we arrive at the Lipatov vertex

$$
\begin{aligned}
\mathscr{A}_{\mathrm{L}}= & -2 g^{2} \frac{1}{\vec{k}_{\perp}^{2}} \frac{1}{\vec{k}_{\perp}^{\prime 2}} \bar{\xi}_{n} T^{a} \frac{\hbar}{2} \xi_{n} \bar{\xi}_{\bar{n}} T^{b} \frac{h}{2} \xi_{\bar{n}} \\
& \times\left(i g f^{a b c}\right)\left(k_{\perp}^{\alpha}+k_{\perp}^{\prime \alpha}-\frac{1}{2} \bar{n}^{\alpha} n \cdot k^{\prime}-\frac{1}{2} n^{\alpha} \bar{n} \cdot k-\frac{n^{\alpha}}{n \cdot k^{\prime}} \vec{k}_{\perp}^{2}-\frac{\bar{n}^{\alpha}}{\bar{n} \cdot k} \vec{k}_{\perp}^{2}\right) .
\end{aligned}
$$

This vertex is gauge invariant, as can be explicitly verified by contracting with the external gluon momentum.

Now we have all the pieces needed to calculate the quark scattering cross section in the forward region. Squaring the amplitude in Eq. (4.1) we obtain the tree level cross section

$$
\sigma^{L O}=\frac{2 \alpha_{s}^{2} C_{F}}{N_{c}} \int \frac{d^{2} \vec{k}_{\perp}^{2}}{\vec{k}_{\perp}^{2}} \int \frac{d^{2} \vec{k}_{\perp}^{\prime 2}}{\vec{k}_{\perp}^{\prime 2}} \delta^{(2)}\left(\vec{k}_{\perp}-\vec{k}_{\perp}^{\prime}\right) .
$$

The NLO virtual corrections give

$$
\begin{aligned}
\sigma_{V}^{N L O}=\frac{2 \alpha_{s}^{2} C_{F}}{N_{c}} \int \frac{d^{2} \vec{k}_{\perp}}{\vec{k}_{\perp}^{2}} \int \frac{d^{2} \vec{k}_{\perp}^{\prime}}{\vec{k}_{\perp}^{\prime 2}} \delta^{(2)}\left(\vec{k}_{\perp}-\vec{k}_{\perp}^{\prime}\right) \\
\quad \times\left(-\frac{\alpha_{s} N_{c}}{2 \pi^{2}}\right) v^{2 \eta} w(v)^{2} \frac{\Gamma(\eta) \Gamma\left(\frac{1}{2}-\eta\right)}{\sqrt{\pi}} \int d^{2} q_{\perp} \frac{\vec{k}_{\perp}^{2}}{\vec{q}_{\perp}^{2}} \frac{1}{\left[\left(\vec{q}_{\perp}-\vec{k}_{\perp}\right)^{2}\right]^{1+\eta}} .
\end{aligned}
$$

The NLO real corrections can be obtained by the standard method of squaring the amplitude and summing over final states, or by taking the cut of the forward scattering graph in the Glauber regime. In order to incorporate the rapidity regulator we use the latter method to obtain

$$
\begin{aligned}
\sigma_{R}^{N L O}=\frac{2 \alpha_{s}^{2} C_{F}}{N_{c}} & \int \frac{d^{2} \vec{k}_{\perp}}{\vec{k}_{\perp}^{2}} \int \frac{d^{2} \vec{k}_{\perp}^{\prime}}{\vec{k}_{\perp}^{\prime 2}} \\
& \times\left(\frac{\alpha_{s} N_{c}}{\pi^{2}}\right) v^{2 \eta} w(v)^{2} \frac{\Gamma(\eta) \Gamma\left(\frac{1}{2}-\eta\right)}{\sqrt{\pi}} \int d^{2} q_{\perp} \frac{\delta^{(2)}\left(\vec{q}_{\perp}-\vec{k}_{\perp}^{\prime}\right)}{\left[\left(\vec{q}_{\perp}-\vec{k}_{\perp}\right)^{2}\right]^{1+\eta}} .
\end{aligned}
$$

In order to ensure that there is no double counting in SCET the soft-Glauber overlap region needs to be subtracted from the above results, however in this case the overlap region vanishes. Adding these up we arrive at an expression for the forward scattering cross section accurate to NLO

$$
\begin{aligned}
\sigma= & \frac{2 \alpha_{s}^{2} C_{F}}{N_{c}} \int \frac{d^{2} \vec{k}_{\perp}}{\vec{k}_{\perp}^{2}} \int \frac{d^{2} \vec{k}_{\perp}}{\vec{k}_{\perp}^{\prime 2}}\left\{\delta^{(2)}\left(\vec{k}_{\perp}-\vec{k}_{\perp}^{\prime}\right)+\left(\frac{\alpha_{s} N_{c}}{\pi^{2}}\right) \frac{\Gamma(\eta) \Gamma\left(\frac{1}{2}-\eta\right)}{\sqrt{\pi}} v^{2 \eta} w(v)^{2}\right. \\
& \left.\times \int \frac{d^{2} q_{\perp}}{\left[\left(\vec{q}_{\perp}-\vec{k}_{\perp}\right)^{2}\right]^{1+\eta}}\left[\delta^{(2)}\left(\vec{q}_{\perp}-\vec{k}_{\perp}^{\prime}\right)-\frac{\vec{k}_{\perp}^{2}}{2 \vec{q}_{\perp}^{2}} \delta^{(2)}\left(\vec{k}_{\perp}-\vec{k}_{\perp}^{\prime}\right)\right]\right\}
\end{aligned}
$$

Expanding around $\eta=0$ we can isolate the rapidity divergent term

$$
\begin{aligned}
\sigma= & \frac{2 \alpha_{s}^{2} C_{F}}{N_{c}} \int \frac{d^{2} \vec{k}_{\perp}}{\vec{k}_{\perp}^{2}} \int \frac{d^{2} \vec{k}_{\perp}}{\vec{k}_{\perp}^{\prime 2}}\left\{\delta^{(2)}\left(\vec{k}_{\perp}-\vec{k}_{\perp}^{\prime}\right)\right. \\
& \left.+\left(\frac{\alpha_{s} N_{c}}{\pi^{2}}\right) \frac{w(v)^{2}}{\eta} \int \frac{d^{2} q_{\perp}}{\left(\vec{q}_{\perp}-\vec{k}_{\perp}\right)^{2}}\left[\delta^{(2)}\left(\vec{q}_{\perp}-\vec{k}_{\perp}^{\prime}\right)-\frac{\vec{k}_{\perp}^{2}}{2 \vec{q}_{\perp}^{2}} \delta^{(2)}\left(\vec{k}_{\perp}-\vec{k}_{\perp}^{\prime}\right)\right]+\ldots\right\}
\end{aligned}
$$


where the dots represent NLO terms that are finite in the $\eta \rightarrow 0$ limit. This result raises the important question of how the rapidity divergence is subtracted. In SCET without Glauber gluons collinear and soft degrees of freedom factor and observables can often be expressed as convolutions of matrix elements of operators involving only collinear or soft degrees of freedom. If the factorization of soft and collinear holds in the presence of Glauber gluons then it may be that the above cross section can also be expressed as a convolution of the matrix element of a soft operator with the matrix element of an $n$-collinear operator and the matrix element of an $\bar{n}$-collinear operator. In this case the counter-term for the soft operator would cancel the rapidity divergence. Such a factorization is suggested by the standard treatment in the literature [35], where the twodimension Dirac delta function in transverse-momentum space is interpreted as the BFKL Green function. The rapidity divergence is then canceled by a counter-term for this Green function. However, factorization of the Glauber interaction in SCET requires an all orders summation of soft gluons, which has not yet been accomplished. A first step in this direction has recently been made in Ref. [38] where it is shown that in a scalar theory with $n$-collinear modes, $\bar{n}$-collinear modes, and Glauber modes an all orders summation of ladder graphs gives the leading Regge behavior. We will leave the summation of soft gluons for a future work, and motivated by the BFKL approach will for the time being conjecture that the cross section factors. We renormalize the rapidity divergence by identifying the two-dimension Dirac delta function in transverse-momentum space as the leading order vacuum matrix element of a (currently unknown) operator, $O_{G}^{\text {soft }}$, involving soft fields: $G\left(\vec{k}_{\perp}-\vec{k}_{\perp}^{\prime}\right) \equiv\left\langle O_{G \text {,soft }}\right\rangle$. Then

$$
\begin{aligned}
G\left(\vec{k}_{\perp}-\vec{k}_{\perp}^{\prime}, v\right) & =\int d^{2} \ell_{\perp} \mathscr{Z}^{-1}\left(\vec{k}_{\perp}-\vec{\ell}_{\perp} ; \eta, v\right) G\left(\vec{\ell}_{\perp}-\vec{k}_{\perp}^{\prime} ; v\right)^{(0)} \\
& =\int d^{2} \ell_{\perp} \mathscr{Z}^{-1}\left(\vec{k}_{\perp}-\vec{\ell}_{\perp} ; \eta, v\right) \delta^{(2)}\left(\vec{\ell}_{\perp}-\vec{k}_{\perp}^{\prime}\right) \\
& =\delta^{(2)}\left(\vec{k}_{\perp}-\vec{k}_{\perp}^{\prime}\right)+\text { counterterms }
\end{aligned}
$$

where the superscript (0) indicates the matrix element of the bare operator. Inverting the above equation leads to

$$
\delta^{(2)}\left(\vec{k}_{\perp}-\vec{k}_{\perp}^{\prime}\right)=\int d^{2} \ell_{\perp} \mathscr{Z}\left(\vec{k}_{\perp}-\vec{\ell}_{\perp} ; \eta, v\right) G\left(\vec{\ell}_{\perp}-\vec{k}_{\perp}^{\prime} ; v\right) .
$$

The rapidity divergence term in Eq. (4.16) is cancelled by setting

$$
\begin{aligned}
\mathscr{Z}\left(\vec{k}_{\perp}-\vec{\ell}_{\perp} ; \eta, v\right)=\delta^{(2)}\left(\vec{k}_{\perp}-\vec{\ell}_{\perp}\right)-\left(\frac{\alpha_{s} N_{c}}{\pi^{2}}\right) \frac{w(v)^{2}}{\eta} & {\left[\frac{1}{\left(\vec{k}_{\perp}-\vec{\ell}_{\perp}\right)^{2}}\right.} \\
& \left.-\frac{1}{2} \delta^{(2)}\left(\vec{k}_{\perp}-\vec{\ell}_{\perp}\right) \int \frac{d^{2} q_{\perp}}{\left(\vec{q}_{\perp}-\vec{k}_{\perp}\right)^{2}} \frac{\vec{k}_{\perp}^{2}}{\vec{q}_{\perp}^{2}}\right] .
\end{aligned}
$$

Inserting this expression into Eq. (4.18) we find

$$
\begin{aligned}
\delta^{(2)}\left(\vec{k}_{\perp}-\vec{k}_{\perp}^{\prime}\right)=G\left(\vec{k}_{\perp}-\vec{k}_{\perp}^{\prime} ; v\right)-\left(\frac{\alpha_{s} N_{c}}{\pi^{2}}\right) \frac{w(v)^{2}}{\eta}\left[\int d^{2} q_{\perp} \frac{G\left(\vec{q}_{\perp}-\vec{k}_{\perp}^{\prime} ; v\right)}{\left(\vec{q}_{\perp}-\vec{k}_{\perp}\right)^{2}}\right. \\
\left.-\frac{1}{2} G\left(\vec{k}_{\perp}-\vec{k}_{\perp}^{\prime} ; v\right) \int \frac{d^{2} q_{\perp}}{\left(\vec{q}_{\perp}-\vec{k}_{\perp}\right)^{2}} \frac{\vec{k}_{\perp}^{2}}{\vec{q}_{\perp}^{2}}\right],
\end{aligned}
$$


which when used in Eq. (4.16) gives

$$
\sigma=\frac{2 \alpha_{s}^{2} C_{F}}{N_{c}} \int \frac{d^{2} \vec{k}_{\perp}}{\vec{k}_{\perp}^{2}} \int \frac{d^{2} \vec{k}_{\perp}^{\prime}}{\vec{k}_{\perp}^{\prime 2}} G\left(\vec{k}_{\perp}-\vec{k}_{\perp}^{\prime} ; v\right)+\ldots
$$

where the singular terms in $\eta$ cancel and the dots indicate NLO terms that do not vanish in the $\eta \rightarrow 0$ limit. The dependence of $G\left(\vec{k}_{\perp}-\vec{k}_{\perp}^{\prime} ; v\right)$ on $v$ is given by the rapidity RGE

$$
\frac{d}{d \ln v} G\left(\vec{k}_{\perp}-\vec{k}_{\perp}^{\prime} ; v\right)=\int d^{2} \ell_{\perp} \gamma_{v}\left(\vec{k}_{\perp}-\vec{\ell}_{\perp}\right) G\left(\vec{\ell}_{\perp}-\vec{k}_{\perp}^{\prime} ; v\right)
$$

where the rapidity anomalous dimension is determined from

$$
\gamma_{v}\left(\vec{k}_{\perp}-\vec{k}_{\perp}^{\prime}\right)=\int d^{2} \ell_{\perp} \mathscr{Z}\left(\vec{\ell}_{\perp},-\vec{k}_{\perp}^{\prime} ; \eta, v\right)^{-1} \frac{d}{d \ln v} \mathscr{Z}\left(\vec{k}_{\perp}-\vec{\ell}_{\perp} ; \eta, v\right) .
$$

Using

$$
\frac{d}{d \ln v}=\frac{\partial}{\partial \ln v}-w(v)^{2} \eta \frac{\partial}{\partial w^{2}}
$$

we find the leading-log (LL) rapidity anomalous dimension

$$
\gamma_{v}\left(\vec{k}_{\perp}-\vec{k}_{\perp}^{\prime}\right)=\left(\frac{\alpha_{s} N_{c}}{\pi^{2}}\right)\left[\frac{1}{\left(\vec{k}_{\perp}-\vec{k}_{\perp}^{\prime}\right)^{2}}-\frac{1}{2} \delta^{(2)}\left(\vec{k}_{\perp}-\vec{k}_{\perp}^{\prime}\right) \int \frac{d^{2} q_{\perp}}{\left(\vec{q}_{\perp}-\vec{k}_{\perp}\right)^{2}} \frac{\vec{k}_{\perp}^{2}}{\vec{q}_{\perp}^{2}}\right],
$$

where we set $w(v)=1$. Using this LL expression in Eq. (4.22) gives

$$
\frac{d}{d \ln v} G\left(\vec{k}_{\perp}-\vec{k}_{\perp}^{\prime} ; v\right)=\left(\frac{\alpha_{s} N_{c}}{\pi^{2}}\right) \int \frac{d^{2} q_{\perp}}{\left(\vec{q}_{\perp}-\vec{k}_{\perp}\right)^{2}}\left[G\left(\vec{q}_{\perp}-\vec{k}_{\perp}^{\prime} ; v\right)-\frac{\vec{k}_{\perp}^{2}}{2 \vec{q}_{\perp}^{2}} G\left(\vec{k}_{\perp}-\vec{k}_{\perp}^{\prime} ; v\right)\right] .
$$

This is the BFKL equation [compare to Eq. (3.58) in Ref. [35]].

\section{Conclusion}

In this talk I have tried to give a compelling motivation for reexamining the contribution of the Glauber region in factorization. In particular, I have shown that Glauber interactions in SCET are responsible for the Regge behavior of the theory, and reproduce the BFKL evolution equation. This is clearly only the beginning. There is much awaiting to be discovered including the connection of mutli-Glauber exchange with saturation physics.

\section{References}

[1] J. C. Collins and D. E. Soper, Ann. Rev. Nucl. Part. Sci. 37, 383 (1987).

[2] G. Sterman, TASI lectures 1995 [arXiv:hep-ph/9606312].

[3] R. L. Jaffe, [arXiv:hep-ph/9602236].

[4] C. W. Bauer, S. Fleming and M. E. Luke, Phys. Rev. D 63, 014006 (2001) [arXiv:hep-ph/0005275].

[5] C. W. Bauer, S. Fleming, D. Pirjol and I. W. Stewart, Phys. Rev. D 63, 114020 (2001) [arXiv:hep-ph/0011336]. 
[6] C. W. Bauer and I. W. Stewart, Phys. Lett. B 516, 134 (2001) [arXiv:hep-ph/0107001].

[7] C. W. Bauer, D. Pirjol and I. W. Stewart, Phys. Rev. D 65, 054022 (2002) [arXiv:hep-ph/0109045].

[8] C. W. Bauer, S. Fleming, D. Pirjol, I. Z. Rothstein and I. W. Stewart, Phys. Rev. D 66, 014017 (2002) [arXiv:hep-ph/0202088].

[9] M. Beneke, A. P. Chapovsky, M. Diehl and T. Feldmann, Nucl. Phys. B 643, 431 (2002) [arXiv:hep-ph/0206152]

[10] M. Beneke and T. Feldmann, Phys. Lett. B 553, 267 (2003) [arXiv:hep-ph/0211358]

[11] C. W. Bauer, D. Pirjol and I. W. Stewart, Phys. Rev. D 67, 071502 (2003). [hep-ph/0211069].

[12] S. Mantry, D. Pirjol and I. W. Stewart, Phys. Rev. D 68, 114009 (2003). [hep-ph/0306254].

[13] R. J. Hill, T. Becher, S. J. Lee and M. Neubert, JHEP 0407, 081 (2004). [hep-ph/0404217].

[14] K. S. M. Lee and I. W. Stewart, Nucl. Phys. B 721, 325 (2005). [hep-ph/0409045].

[15] S. W. Bosch, M. Neubert and G. Paz, JHEP 0411, 073 (2004). [hep-ph/0409115].

[16] C. Marcantonini and I. W. Stewart, Phys. Rev. D 79, 065028 (2009). [arXiv:0809.1093 [hep-ph]].

[17] D. W. Kolodrubetz, I. Moult and I. W. Stewart, [arXiv:1601.02607 [hep-ph]].

[18] L. D. Landau, Nucl. Phys. 13, 181 (1959).

[19] S. Coleman and R. E. Norton, Nuovo Cim. 38, 438 (1965).

[20] J. C. Collins and G. F. Sterman, Nucl. Phys. B 185, 172 (1981).

[21] G. T. Bodwin, S. J. Brodsky and G. P. Lepage, Phys. Rev. Lett. 47, 1799 (1981).

[22] J. C. Collins, D. E. Soper and G. F. Sterman, Phys. Lett. B 109, 388 (1982).

[23] G. T. Bodwin, Phys. Rev. D 31, 2616 (1985), [Phys. Rev. D 34, 3932 (1986)].

[24] J. C. Collins, D. E. Soper and G. F. Sterman, Nucl. Phys. B 261, 104 (1985).

[25] S. M. Aybat and G. F. Sterman, Phys. Lett. B 671, 46 (2009). [arXiv:0811.0246 [hep-ph]].

[26] F. Liu and J. P. Ma, [arXiv:0802.2973 [hep-ph]].

[27] C. W. Bauer, B. O. Lange and G. Ovanesyan, JHEP 1107, 077 (2011). [arXiv:1010.1027 [hep-ph]].

[28] A. Idilbi and A. Majumder, Phys. Rev. D 80, 054022 (2009). [arXiv:0808.1087 [hep-ph]].

[29] F. D’Eramo, H. Liu and K. Rajagopal, Phys. Rev. D 84, 065015 (2011). [arXiv:1006.1367 [hep-ph]].

[30] M. Benzke, N. Brambilla, M. A. Escobedo and A. Vairo, JHEP 1302, 129 (2013). [arXiv:1208.4253 [hep-ph]].

[31] J. F. Donoghue and D. Wyler, Phys. Rev. D 81, 114023 (2010). [arXiv:0908.4559 [hep-ph]].

[32] J. F. Donoghue, PoS EFT 09, 001 (2009). [arXiv:0909.0021 [hep-ph]].

[33] V. S. Fadin, E. A. Kuraev and L. N. Lipatov, Phys. Lett. B 60, 50 (1975).

[34] I. I. Balitsky and L. N. Lipatov, Sov. J. Nucl. Phys. 28, 822 (1978), [Yad. Fiz. 28, 1597 (1978)].

[35] Y. V. Kovchegov and E. Levin, Quantum chromodynamics at high energy (Cambridge University Press, The Edinburgh Building, Cambridge CB2 8RU, UK, 2012), 1st ed. 
[36] I. W. Stewart and I. Z. Rothstein, (work in progress). Talk "An effective field theory for forward scattering and factorization violation" presented by I. Z. Rothstein at SCET2015 in Santa Fe, NM, URL https://indico.fnal.gov/conferenceDisplay.py?confId=9397

[37] S. Fleming, Phys. Lett. B 735, 266 (2014). [arXiv:1404.5672 [hep-ph]].

[38] J. F. Donoghue, B. K. El-Menoufi and G. Ovanesyan, Phys. Rev. D 90, no. 9, 096009 (2014). [arXiv:1405.1731 [hep-ph]].

[39] M. J. Dugan and B. Grinstein, Phys. Lett. B 255, 583 (1991).

[40] A. V. Manohar, T. Mehen, D. Pirjol and I. W. Stewart, Phys. Lett. B 539, 59 (2002) [arXiv:hep-ph/0204229].

[41] J. g. Chay and C. Kim, arXiv:hep-ph/0205117.

[42] A. V. Manohar and I. W. Stewart, Phys. Rev. D 76, 074002 (2007) doi:10.1103/PhysRevD.76.074002 [hep-ph/0605001].

[43] J. Y. Chiu, A. Jain, D. Neill and I. Z. Rothstein, JHEP 1205, 084 (2012) doi:10.1007/JHEP05(2012)084 [arXiv:1202.0814 [hep-ph]].

[44] J. y. Chiu, A. Jain, D. Neill and I. Z. Rothstein, Phys. Rev. Lett. 108, 151601 (2012) doi:10.1103/PhysRevLett.108.151601 [arXiv:1104.0881 [hep-ph]].

[45] J. R. Gaunt, JHEP 1407, 110 (2014) doi:10.1007/JHEP07(2014)110 [arXiv:1405.2080 [hep-ph]].

[46] M. Grazzini, A. Papaefstathiou, J. M. Smillie and B. R. Webber, JHEP 1409, 056 (2014) doi:10.1007/JHEP09(2014)056 [arXiv:1403.3394 [hep-ph]].

[47] I. W. Stewart and I. Z. Rothstein, (work in progress). Talk "Glauber Gluons in SCET" presented by I. W. Stewart at SCET2010 in Ringberg, Germany, URL https://indico.mpp.mpg.de/conferenceDisplay.py?confId=632 